\title{
Meeting Report of the Fifth International Cancer Epigenetics Conference in Beijing, China, October 2016
}

\section{Fifth International Cancer Epigenetics Conference}

Beijing, China, 21-23 October 2016

This meeting reported many new findings in the field of cancer epigenetics, including basic science, translational and clinical studies. In this report, we summarize some of the main advancements and prospects in cancer epigenetics presented at this meeting.

First draft submitted: 25 February 2017; Accepted for publication: 26 April 2017; Published online: 22 May 2017

Keywords: $5 \mathrm{hmc} \bullet$ DNA methylation $\bullet$ histone modification $\bullet$ imprinting $\bullet$ noncoding RNA - RNA methylation

Cancer epigenetics has become an important field in oncology since the discovery of aberrant DNA methylation in colorectal cancer in 1983 [1]. This field was further promoted by the development of methylation-specific PCR (MSP) in 1996 [2]. Compared with gene mutations, epigenetic alterations are observed more frequently, and they usually occur in a cell type-specific or individualized manner. Cancer epigenetics is becoming an important part of precision medicine [3], and it has been applied to early cancer detection, prognosis and therapeutic prediction.

The honorary president, James G Herman (University of Pittsburgh Cancer, PA, USA) welcomed participants and acknowledged the scientific committee. A series of programs were arranged within the field of cancer epigenetics, including DNA methylation, imprinting, histone modification and noncoding RNA. In these 3 days, basic and translational studies as well as clinical applications, were discussed by leading scientists from the USA, Europe and Asia.

\section{DNA methylation \& reprogramming}

The paradigm of DNA methylation pat- terns was described as de novo methylation by DNMT3a/3b and maintenance methylation by DNMT1. The established model was challenged by a finding from Zhibing Wang's lab (Johns Hopkins University, MD, USA). Their results suggested that all three DNMTs were needed to maintain CG methylation symmetry. They also found that retrotransposon long terminal repeats were specifically methylated by DNMT1, while long interspersed nuclear elements were mainly methylated by DNMT3a/3b. Dr Wang's group method, MethylMosaic. Using this method, 7 novel differentially methylated regions from known loci and 20 novel differentially methylated regions from unknown loci were found. These novel and known imprinted loci were vulnerable to bisphenol A exposure.

DNA methylation is a reversible process, through either passive or active demethylation. Passive demethylation has been well documented and attributed to reduction in activities or absence of DNMTs during DNA replication. The recently discovered 5-hydroxymethylcytosine $(5 \mathrm{hmC})$ in the mammalian genome is associated with DNA established a novel imprinting detection
Dan Gao',2, James G Herman ${ }^{3}$, Hengmi Cui ${ }^{4}$, Jin Jen ${ }^{5}$, Francois Fuks ${ }^{6}$, Malcolm V Brock ${ }^{7}$, Toshikazu Ushijima ${ }^{8}$, Carlo Croce ${ }^{9}$, Yoshimitsu Akiyama $^{10}$ \& Mingzhou Guo*,1 'Department of Gastroenterology \& Hepatology, Chinese PLA General Hospital, Beijing 100853, China ${ }^{2}$ School of Medicine, Nankai University, Tianjin 300071, China

${ }^{3}$ Department of Medicine, University of Pittsburgh School of Medicine, Pittsburg, PA 15232, USA

${ }^{4}$ Institute of Epigenetics \& Epigenomics, Yangzhou University, Yangzhou 225000, China

${ }^{5}$ Department of Laboratory of Medicine \& Pathology, Mayo Clinic, Rochester, MN 55905, USA

${ }^{6}$ Laboratory of Cancer Epigenetics, Faculty of Medicine, ULB Cancer Research Center (U-CRC), Université Libre de Bruxelles (ULB), Brussels 1070 , Belgium

${ }^{7}$ Oncology Center, Johns Hopkins University, Baltimore, MD 21231, USA ${ }^{8}$ Division of Epigenomics, National Cancer Center Research Institute, Tokyo 104-0045, Japan

${ }^{9}$ Department of Cancer Biology \& Genetics, College of Medicine, The Ohio State University Comprehensive Cancer Center, Columbus, OH 43210, USA ${ }^{10}$ Department of Molecular Oncology, Graduate School of Medical \& Dental Sciences, Tokyo Medical \& Dental University, Tokyo 113-8519, Japan *Author for correspondence:

Tel.: +861066937651

Fax: +861068180325 mzguo@hotmail.com

\section{Future : Medicine part of}


demethylation and is induced by oxidizing 5-methylcytosine $(5 \mathrm{mC})$ to $5 \mathrm{hmC}$. This dynamic change is designated as 'active demethylation' [4]. Jiang Liu (Chinese Academy of Sciences, Beijing, China) reported that sperm DNA methylome was inherited in the early stage of zebrafish development, while in early embryos of mammals, the reprogramming of paternal methylome and a proportion of maternal methylome were achieved by active demethylation. The ten-eleven translocation (TET) family of DNA hydroxylases, including TET1, TET2 and TET3, mediates the conversion of $5 \mathrm{mC}$ to $5 \mathrm{hmC}$. Inactive mutations or deletions of TET 2 were frequently detected in hematopoietic malignancies, along with decreased $5 \mathrm{hmC}$ levels, while no somatic mutations were found in TET1 or TET3 $[5,6]$. Qian Tao (The Chinese University of Hong Kong, China) discovered that TET1 was frequently methylated in various tumors, and the expression of TET1 was regulated by promoter methylation. TET-mediated $5 \mathrm{mC}$ oxidation to $5 \mathrm{hmC}$ requires co-substrates, such as 2-ketoglutarate, which is mainly generated by isocitrate dehydrogenase [7]. Weimin $\mathrm{Ci}$ (Chinese Academy of Sciences, China) found that global $5 \mathrm{mC}$ levels did not significantly change during kidney tumorigenesis. Global loss of $5 \mathrm{hmC}$ in kidney tumor tissues was associated with shorter overall survival. Downregulation of IDH1 is one of the mechanisms underlying $5 \mathrm{hmC}$ loss in kidney cancer [8].

\section{DNA methylation in cancer detection, prognosis, prediction \& treatment}

James G Herman recently developed a new DNA methylation detection method, methylation-on-beads (MOB). MOB increased sensitivity by reducing sample loss. By using $\mathrm{MOB}$ and real-time quantitative MSP, genes frequently methylated in lung cancer $(S O X$ 17, TAC1, HOXA7, CDO1, HOXA9 and ZFP42) were screened. This study indicated that high sensitivity and specificity of detection for early-stage non-smallcell lung cancer could be achieved using a panel of promoter-methylated genes in plasma (CDO1, TAC1 and SOX17) and sputum (TAC1, HOXA17 and SOX17). In addition, the methylation level of these genes was associated with a high lung cancer risk independent of age, pack-year and nodule size. Evaluating 152 candidate methylation genes by quantitative MSP in lung cancer cell lines and a pilot set of lung tissues, Jin Jen's group (Mayo Clinic, NY, USA) identified a panel of highly sensitive and specific methylation markers (DMRTA, HOXA9, ZIC4, HOXA7 and $S I X 3)$ that can be used to improve early lung cancer detection and monitoring [9]. Jingyi Li (Peking University, China) reported on a new methylation detection method, methylated $\mathrm{CpG}$ tandems amplification and sequencing, which can detect thousands of hypermethylated $\mathrm{CpG}$ islands simultaneously in circulating cell-free DNA. By analyzing a cohort of tissue and plasma samples $(n=151)$ from hepatocellular carcinoma patients and control subjects, a set of classifiers including 19 specific methylation markers achieved a sensitivity of $94 \%$ with a specificity of $89 \%$. Notably, all $15 \alpha$-fetoprotein-negative hepatocellular carcinoma patients were identified by this method.

Toshikazu Ushijima's group (National Cancer Center Research Institute, Tokyo, Japan) found that gastric chronic inflammation triggered by Helicobacter pylori infection did not increase the expression of DNMTs, but decreased the expression of TET2 and TET3 via upregulation of specific miRNA genes. In a prospective cohort study, DNA methylation levels of three preselected marker genes (miR-124a-3, EMX1 and NKX6-1) were measured in noncancerous gastric tissues of 826 endoscopically treated gastric cancer (GC) patients. After following up $826 \mathrm{GC}$ patients for 5 years, 133 patients developed metachronous GCs. Cox proportional hazard regression model analysis indicated that these epigenetic markers were predictive of cancer risk [10]. Joo-Mi Yi (Dongnam Institute of Radiological \& Medical Sciences, Busan, Korea) found that the NEURL gene was frequently methylated in colorectal cancer, and methylation of NEURL upregulated Wnt/ $\beta$-catenin signaling. Methylation of NEURL was associated with overall survival and recurrence-free survival in colorectal cancer, probably methylation of NEURL sensitized chemotherapy. Malcolm V Brock (Johns Hopkins University, MD, USA) reported on combined epigenetic therapy and chemotherapy/immunotherapy in human lung cancer and mouse models. Low doses of azacitidine (AZA) treatment induced reversal of chemotherapy resistance (carboplatin, Taxol, Avastin) in advanced stage IV lung cancer patients. AZA-based therapy in approximately 100 patients with chemorefractory, advanced stage IV lung cancer patients showed $23 \%$ disease stabilization versus 3\% objective response. Low doses of AZA and entinostat decreased the percentage of myeloid-derived Ly $6 \mathrm{G}^{+}$cells and increased the percentage of dendritic cells and macrophages both in vitro and in vivo in Lewis lung carcinoma mice. Weidong Han (Chinese PLA General Hospital, Beijing, China) reported that low-dose decitabine enhanced cytotoxicity of cisplatin in colorectal, breast, esophageal and ovarian cancer cells. Low-dose decitabine targeted cancer stem cells via miRNA-497 in colorectal cancer.

Long-Cheng Li (Peking Union Medical College Hospital of Chinese Academy of Medical Sciences, Beijing, China) introduced MethPrimer 2.0 [11], which is an upgraded version of the popular MethPrimer online 
tool for designing PCR primers for DNA methylation mapping.

\section{Aberrant histone modifications in cancer}

Recently, a high frequency of mutations in histoneencoding genes, rather than histone regulators, has been identified in a number of cancer types. Notably, these mutations are located at or adjacent to $\mathrm{H} 3$ lysine residues that undergo acetylation and/or methylation. For example, the H3K36M mutation is found to occur predominantly in chondroblastomas [12] and rarely in other cancer types. SETD2 serves as the major H3K36 methyltransferase. Haitao Li (Tsinghua University, Beijing, China) reported the crystal structures of the SETD2 catalytic domain bound to H3K36M/I peptides and $S$-adenosylhomocysteine. The catalytic domain adopted an open conformation with the $\mathrm{K} 36 \mathrm{M} / \mathrm{I}$ peptide securely positioned in a newly formed substrate channel. The enzymatic activity of SETD2 was severely reduced.

EZH2 is a key epigenetic regulator that catalyzes the trimethylation of H3K27. Hongquan Zhang (Peking University, Beijing, China) reported that EZH2 was acetylated by P300/CBP associated factor acetyltransferase and deacetylated by SIRT1 deacetylase. P300/CBP associated factor interacted with and acetylated EZH2 mainly at lysine 348 (K348). K348 acetylation decreased EZH2 phosphorylation at T345 and T487 and increased EZH2 stability without disrupting the formation of polycomb repressive complex 2 . EZH2 K348 acetylation was a poor prognosis marker in lung adenocarcinoma. Yoshimitsu Akiyama (Tokyo Medical and Dental University, Tokyo, Japan) found that the expression of SET7/9, a histone monomethyltransferase, was frequently reduced in human GC, and reduced SET7/9 expression was significantly associated with worse prognosis of GC patients. SET7/9 may serve as a tumor suppressor. SETDB2, a histone H3K9 trimethyltransferase, was highly expressed in primary GCs, which was associated with worse prognosis of GC patients. SETDB2 was recruited to the promoter regions of WWOX and CADMI and trimethylated $\mathrm{H} 3 \mathrm{~K}$, resulting in transcriptional repression of these genes. SETDB2 was an oncogenic gene in GC. Specific inhibitors targeting SETDB2 were also developed, and effective inhibition of gastric cancer cell growth was validated.

Jingxuan Pan (Sun Yat-Sen University, Guangzhou, China) reported that PRMT5 was overexpressed in human chronic myelogenous leukemia (CML) stem cells. PRMT5 knockdown or PJ-68 (a novel small-molecule inhibitor of PRMT5 methyltransferase activity) treatment dramatically prolonged survival of retroviral BCR-ABL driven CML mice and impaired in vivo self- renewal capacity of CML stem cells [13]. PRMT5 may represent a novel therapeutic target against leukemic stem cells.

Qing Miao's group (Medical College of Wisconsin, WI, USA) quantified 36 histone variants by SILACbased proteomic analysis, and 150 differentially expressed histone peptides were identified in breast cancer cells treated with estrogen. Proteomics is a powerful tool to reveal histone protein modification. Mingzhou Guo's group (Chinese PLA General Hospital, Beijing, China) found that $\mathrm{C}_{60}(\mathrm{OH})_{22}$ (a nanoparticle) was a potential histone deacetylase inhibitor. $\mathrm{C}_{60}(\mathrm{OH})_{22}$ suppressed angiogenesis in human umbilical vein endothelial cells xenograft mice by inhibiting HDAC1 and HDAC2.

\section{Noncoding RNA in cancer}

In the past two decades, miRNA has become a very hot topic. The alterations of miRNA expression contribute to the pathogenesis of various human malignancies. Carlo Croce (The Ohio State University, OH, USA), a keynote speaker, reviewed the causes and consequences of miRNA dysregulation in cancer and pointed out that future targeted miRNA therapy will depend on identifying all of the targets and pathways of miRNAs involved in cancer and establishing their contribution to malignant transformation, since miRNAs have multiple targets and duplexing functions. Wenqiang Yu (Fudan University, Shanghai, China) identified a subset of miRNAs that functioned as universal and natural gene activators emanating from enhancer loci, termed nuclear activating miRNA (NamiRNA). These miRNAs were associated with active enhancers characterized by distinct H3K27ac enrichment, p300/CBP binding and DNase I hypersensitivity. Ectopic expression of NamiRNA led to the transcriptional activation of neighboring genes. NamiRNA promoted genomewide gene transcription through the binding and activation of its targeted enhancers.

Noncoding RNAs more than 200 bases in length are known as long noncoding RNAs (lncRNAs). LncRNAs have emerged as important regulators of cellular functions and tumorigenesis. Runsheng Chen's group (Chinese Academy of Sciences, Beijing, China) developed a database for prediction of IncRNAs called NONCODE [14]. Recently, they developed another database, NPInter [15], which collected interactions between ncRNAs and other biomolecules (proteins, mRNAs, miRNA or genomic DNAs). They also iden-

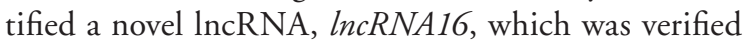
as a potential lung cancer early detection marker and a possible therapeutic target.

Endogenous retroviruses (ERVs) are an important component of the genome and account for approxi- 
mately $8 \%$ of the human genome. Hengmi Cui's group (Yangzhou University, Yangzhou, China) found that one ERV, ALVE1, can transcribe many lncRNAs, which resulted in antiviral effects in vitro through activating an innate immunity pathway. These transcripts were highly expressed in some chicken tissues at early life stage, but they dramatically decreased after 35 days. Pyrosequencing analysis indicated that the expression of this ERV was strictly controlled by DNA methylation.

Xu Song (Sichuan University, Chengdu, China) reported that TMSIAS, a lncRNA transcribed from the opposite strand on the TMS1 gene locus, served as an epigenetic regulator of TMS1 in breast cancer cells. TMSIAS retained within the nuclear physically bind to DNMT1 and G9a, and its silencing led to an impaired occupancy of DNMT1 and G9a at the TMS1 promoter region that thereby modulated $\mathrm{CpG}$ methylation and histone $\mathrm{H} 3 \mathrm{~K} 9 \mathrm{me} 2$ modification. In addition, the cytoplasmic fraction of TMS1AS was found to regulate TMS1 at the translational level by forming an intermolecular duplex with TMS1 mRNA, which influenced the formation of polysomes.

Jingyuan Fang (Shanghai Jiao Tong University School, Shanghai, China) reported that a novel IncRNA, GClncl, served as a scaffold of WDR5 (a key component of histone methyltransferase complex) and KAT2A histone acetyltransferase [16]. The expression of $G C \ln c 1$ was associated with poor prognosis in gastric cancer. Mingzhou Guo's group identified a novel lncRNA, $\operatorname{lncRNA-R54.} \operatorname{LncRNA-R54}$ was highly expressed in $90.3 \%$ of colorectal cancer samples. LncRNA-R54 promoted colorectal cancer development both in vitro and in vivo, suggesting that $\ln c R N A-R 54$ was a novel oncogenic noncoding RNA. Francois Fuks (Free University of Brussels, Brussels, Belgium) reported a genome-wide transcriptional study on the lncRNA expression profile in human breast cancer and identified approximately 300 lncRNAs, which were possible prognostic markers of relapse risk. Guangbiao Zhou (Chinese Academy of Sciences, Beijing, China) identified a novel lncRNA, CAR10 in air pollution related lung cancer. High concentrations of polycyclic aromatic hydrocarbon existed in PM2.5. Multivariate regression analysis showed significant association between CAR10 overexpression and air pollution. The expression of CAR10 was induced by FoxF2 under dibenz $[\mathrm{a}, \mathrm{h}]$ anthracene treatment. CAR1O promoted lung cancer cell growth by upregulation of the oncoprotein EGFR through binding and stabilizing the transcription factor Y-box-binding protein 1.

Recently, an emerging realm of biological regulation, termed epitranscriptomics, revolutionized our understanding of the extent and diversity of mRNA modifications [17]. Hydroxymethylcytosine, well described in DNA, also occurs in RNA, named hmrC and deposited by Tet. Francois Fuks's group conducted a study addressing the distribution, localization and function of cytosine hydroxymethylation in RNA using Drosophila melanogaster as a model. They mapped the transcriptome-wide hydroxymethylation landscape and identified consensus sites for hydroxymethylation. They found that RNA hydroxymethylation can favor mRNA translation. Tet and hydroxymethylated RNA were found to be most abundant in the Drosophila brain, and Tetdeficient fruit flies suffered impaired brain development accompanied by decreased RNA hydroxymethylation. NSUN2 is an m5C methyltransferase that has been shown to catalyze the $\mathrm{m} 5 \mathrm{C}$ modification of eukaryotic RNA [18]. Hengmi Cui's group established an NSUN21. HEK293 cell line through CRISPR-Cas9 mediated homologous recombination repair system. Deficiency of NSUN2 decreased cell proliferation and migration, and influenced the expression of CD44 and MYC.

\section{Acknowledgements}

We thank all the presenters who gave permission for their research to be reported here. Please see their e-mails listed below. We have summarized some of the key ideas that were presented at the meeting and apologize if we have unintentionally omitted any critical aspects. There were many interesting presentations, and we regret that we could not include all of them due to space constraints. James G Herman: hermanj3@upmc.edu; Zhibing Wang: zwang47@jhu. edu; Jiang Liu: liuj@big.ac.cn; Qian Tao: qtao@cuhk.edu. hk; Weimin Ci: ciwm@big.ac.cn; Jin Jen: jen.jin@mayo. edu; Longcheng li: lilongcheng@pumch.ac.cn; Jingyi Li: lijy201@163.com; Toshikazu Ushijima: tushijim@ncc.go.jp; Joo-Mi Yi: jmyi@dirams.re.kr; Malcolm V Brock: mabrock@ jhmi.edu; Weidong Han: hanwdrsw69@yahoo.com; Haitao Li: Iht@tsinghua.edu.cn; Hongquan Zhang: Hongquan. Zhang@bjmu.edu.cn; Yoshimitsu Akiyama: yakiyama. monc@tmd.ac.jp; Jingxuan Pan: panjx2@mail.sysu.edu.cn; Qing Miao: qmiao@mcw.edu; Carlo Croce: carlo.croce@ osumc.edu; Wenqiang Yu: WenqiangYu@fudan.edu.cn; Runsheng Chen: crs@sun5.ibp.ac.cn; Hengmi Cui: hmcui@ yzu.edu.cn; Xu Song: xusong@scu.edu.cn; Jingyuan Fan: jingyuanfang@yahoo.com; Francois Fuks: ffuks@ulb.ac.be; Guangbiao Zhou: guangbiaozhou@163.com

\section{Financial \& competing interests disclosure}

This meeting was supported by grants from the National Basic Research Program of China (973 Program No. 2012CB934002); National Key Scientific instrument Special Programme of China (Grant No.2011YQ03013405); National Science Foundation of China (NSFC No.81672318, 
U1604281, 8167100001, 81402345, 81230047, 81490753, 81121004). Beijing Science Foundation of China (BJSFC No.7171008). Translational foundation of Chinese PLA General Hospital (2016ZHJJ-MS-GMZ). The authors have no other relevant affiliations or financial involvement with any

\section{References}

1 Feinberg AP, Vogelstein B. Hypomethylation distinguishes genes of some human cancers from their normal counterparts. Nature 301(5895), 89-92 (1983).

2 Herman JG, Graff JR, Myohanen S, Nelkin BD, Baylin SB. Methylation-specific PCR: a novel PCR assay for methylation status of CpG islands. Proc. Natl Acad. Sci. USA 93(18), 9821-9826 (1996).

3 Yan W, Guo M. Epigenetics of colorectal cancer. Methods Mol. Biol. 1238, 405-424 (2015).

4 Tahiliani M, Koh KP, Shen Y et al. Conversion of 5-methylcytosine to 5-hydroxymethylcytosine in mammalian DNA by MLL partner TET1. Science 324(5929), 930-935 (2009).

5 Delhommeau F, Dupont S, Della Valle V et al. Mutation in TET2 in myeloid cancers. N. Engl. J. Med.360(22), 2289-2301 (2009).

$6 \mathrm{Li} \mathrm{L}, \mathrm{Li} \mathrm{C}, \mathrm{Mao} \mathrm{H}$ et al. Epigenetic inactivation of the $\mathrm{CpG}$ demethylase TET1 as a DNA methylation feedback loop in human cancers. Sci. Rep. 6, 26591 (2016).

7 Figueroa ME, Abdel-Wahab O, Lu C et al. Leukemic IDH1 and IDH2 mutations result in a hypermethylation phenotype, disrupt TET2 function, and impair hematopoietic differentiation. Cancer Cell 18(6), 553-567 (2010).

8 Chen K, Zhang J, Guo Z et al. Loss of 5-hydroxymethylcytosine is linked to gene body hypermethylation in kidney cancer. Cell Res. 26(1), 103-118 (2016).

9 Bettio D, Venci A, Cariboni U, Di Rocco M, Infante M. Fluorescent in situ hybridization (FISH) in the differential organization or entity with a financial interest in or financial conflict with the subject matter or materials discussed in the manuscript apart from those disclosed.

No writing assistance was utilized in the production of this manuscript.

diagnosis of ground-glass opacities in the lung. Lung Cancer 71(3), 319-322 (2011).

10 Maeda M, Nakajima T, Oda I et al. High impact of methylation accumulation on metachronous gastric cancer: 5-year follow-up of a multicentre prospective cohort study. Gut doi:10.1136/gutjnl-2016-313387 (2016) (Epub ahead of print).

11 MethPrimer 2.0. http://www.urogene.org/methprimer2

12 Behjati S, Tarpey PS, Presneau N et al. Distinct H3F3A and $\mathrm{H} 3 \mathrm{~F} 3 \mathrm{~B}$ driver mutations define chondroblastoma and giant cell tumor of bone. Nat. Genet. 45(12), 1479-1482 (2013).

13 Jin Y, Zhou J, Xu F et al. Targeting methyltransferase PRMT5 eliminates leukemia stem cells in chronic myelogenous leukemia. J. Clin. Invest. 126(10), 3961-3980 (2016).

14 NONCODE. http://www.noncode.org/

15 NPInter. http://www.bioinfo.org/NPInter/

16 Sun TT, He J, Liang Q et al. LncRNA GClnc1 promotes gastric carcinogenesis and may act as a modular scaffold of WDR5 and KAT2A complexes to specify the histone modification pattern. Cancer Discov. 6(7), 784-801 (2016).

17 Gilbert WV, Bell TA, Schaening C. Messenger RNA modifications: form, distribution, and function. Science 352(6292), 1408-1412 (2016).

18 Sakita-Suto S, Kanda A, Suzuki F, Sato S, Takata T, Tatsuka M. Aurora-B regulates RNA methyltransferase NSUN2. Mol. Biol. Cell 18(3), 1107-1117 (2007). 